Phys. Rev. D 92, 025007 (2015)

arXiv:1504.01324

\title{
Parton-model calculation of a nonstandard decay process in isotropic modified Maxwell theory
}

\author{
J.S. Díaz* and F.R. Klinkhamer ${ }^{\dagger}$ \\ Institute for Theoretical Physics, \\ Karlsruhe Institute of Technology (KIT), \\ 76128 Karlsruhe, Germany
}

\begin{abstract}
We have performed a calculation in the parton-model approximation of a nonstandard decay process in isotropic modified Maxwell theory coupled to standard Dirac theory, with a single Lorentz-violating parameter $\kappa$ in the photonic sector. Previous calculations of this process (vacuum Cherenkov radiation by a proton for the theory with $\kappa>0$ ) were performed for pointlike particles, and an upper bound on $\kappa$ at the $10^{-19}$ level was obtained from experimental data on ultra-high-energy cosmic rays. The parton-model results change the decay rate by about an order of magnitude but give the same upper bound on $\kappa$ because the threshold energy is unchanged. The previous point-particle calculation of photon decay into an electron-positron pair for the theory with $\kappa<0$ remains valid, and experimental results on cosmic gamma rays provide a lower bound on $\kappa$ at the $-10^{-15}$ level.
\end{abstract}

PACS numbers: 11.30.Cp, 12.20.-m, 98.70.Rz, 98.70.Sa

Keywords: Lorentz violation, quantum electrodynamics, gamma-ray sources, cosmic rays

\footnotetext{
* jorge.diaz@kit.edu

$\dagger$ frans.klinkhamer@kit.edu
} 


\section{INTRODUCTION}

Possible small deviations of Lorentz invariance in the photonic sector are best bounded by direct experiments in the laboratory (references will be given later). But it is also possible to obtain tight indirect bounds on the Lorentz violation by detecting charged or neutral particles with ultrahigh energies in the Earth's atmosphere, possibly coming from distant astronomical sources.

For these indirect but terrestrial bounds, it is of crucial importance to determine the theoretical decay rates reliably. Up till now, most of these calculations were performed for pointlike particles. The pointlike calculations are expected to be reliable for certain decay processes (e.g., photon decay into an electron-positron pair if kinematically allowed) but not for other decay processes (e.g., vacuum Cherenkov radiation by protons or heavy nuclei if kinematically allowed). These latter processes are better calculated using the parton-model approximation and the present article sets out to perform such a calculation for vacuum Cherenkov radiation by a realistic proton.

\section{BACKGROUND}

Throughout this article, we use natural units with $\hbar=1=c$ and the standard Minkowski metric $g_{\mu \nu}(x)=\eta_{\mu \nu} \equiv[\operatorname{diag}(+1,-1,-1,-1)]_{\mu \nu}$. As the theory considered will be seen to violate Lorentz invariance, we must state clearly what is meant by $c$. For the particular theory considered, $c$ is the maximum attainable velocity of the fermionic particles.

The Lagrange density for modified electrodynamics in the presence of CPT-even Lorentz violation in the photon sector can be written as follows:

$$
\mathcal{L}_{\text {modMaxwell }}=-\frac{1}{4} \eta_{\mu \rho} \eta_{\nu \sigma} F^{\mu \nu} F^{\rho \sigma}-\frac{1}{4}\left(k_{F}\right)_{\mu \nu \rho \sigma} F^{\mu \nu} F^{\rho \sigma}
$$

in terms of the conventional field strength tensor $F^{\mu \nu}=\partial^{\mu} A^{\nu}-\partial^{\nu} A^{\mu}$. The first term on the right-hand side of (2.1) describes standard Maxwell electrodynamics and the second term corresponds to a dimension-four operator that breaks Lorentz invariance [1-3]. The size of the deviation from exact Lorentz invariance is controlled by the dimensionless coefficient $\left(k_{F}\right)_{\mu \nu \rho \sigma}$. The Lagrange density (2.1) describes a theory which preserves CPT, gauge, and coordinate invariance, whereas particle Lorentz invariance is broken. The coefficient $\left(k_{F}\right)_{\mu \nu \rho \sigma}$ has the symmetries of the Riemann tensor and has 20 independent components. Its double trace can be taken to be zero because it simply modifies the normalization of the Lorentzinvariant part of the Lagrange density (2.1). In other words, the coefficient $k_{F}$ is assumed to satisfy $\left(k_{F}\right)_{\mu \nu}^{\mu \nu}=0$, which reduces the total number of independent components to 19 .

The modified field equations are given by

$$
\partial^{\alpha} F_{\alpha \beta}+\left(k_{F}\right)_{\alpha \beta \mu \nu} \partial^{\alpha} F^{\mu \nu}=0 .
$$

From (2.2), it can be seen that ten of the 19 independent components of the coefficient $\left(k_{F}\right)_{\alpha \beta \mu \nu}$ produce birefringence in the photon sector, which can be bounded with remarkable precision using cosmological observations [4-6]. For this reason, we focus in the present article on the remaining nine components that produce nonbirefringent effects. If the ten components generating birefringence are neglected, the coefficient $\left(k_{F}\right)_{\alpha \beta \mu \nu}$ can be expressed 
as follows [3]

$$
\begin{aligned}
\left(k_{F}\right)_{\alpha \beta \mu \nu}=\frac{1}{2}[ & \eta_{\alpha \mu}\left(k_{F}\right)_{\beta \lambda \nu}^{\lambda}-\eta_{\alpha \nu}\left(k_{F}\right)_{\beta \lambda \mu}^{\lambda} \\
& \left.-\eta_{\beta \mu}\left(k_{F}\right)^{\lambda}{ }_{\alpha \lambda \nu}+\eta_{\beta \nu}\left(k_{F}\right)^{\lambda}{ }_{\alpha \lambda \mu}\right],
\end{aligned}
$$

where $\left(k_{F}\right)^{\lambda}{ }_{\mu \lambda \nu}$ is traceless and symmetric in the indices $\mu$ and $\nu$. A simple form for this nonbirefringent coefficient can be obtained if we restrict the modified Maxwell theory to describe an isotropic deviation from exact Lorentz invariance. For this isotropic case, the relevant coefficient in the last expression of (2.1) can be written as (2.3) with

$$
\left(k_{F}\right)^{\lambda}{ }_{\mu \lambda \nu}=\frac{\kappa}{2}[\operatorname{diag}(3,1,1,1)]_{\mu \nu},
$$

where $\kappa$ is a short-hand version of $\widetilde{\kappa}_{\text {tr }}$ used in most of the recent literature.

The isotropic breakdown of Lorentz invariance in the photonic sector is then controlled by the single coupling constant $\kappa$ which physically parameterizes a modification of the photon phase velocity. Modern versions of the Ives-Stilwell experiment [7] allow for direct laboratory bounds on this parameter down to the $10^{-10}$ level [8-10].

The parameter $\kappa$ endows the vacuum with an effective index of refraction

$$
n=\sqrt{(1+\kappa) /(1-\kappa)} .
$$

Writing the photon momentum in the form $q^{\mu}=(\omega(q), q, 0,0)$ for $q \equiv|\boldsymbol{q}|$, the photon energy and momentum are related by

$$
\omega(q)=\frac{1}{n} q=\sqrt{\frac{1-\kappa}{1+\kappa}} q .
$$

It will be seen that this modified photon dispersion relation allows for processes which are kinematically forbidden in the conventional Lorentz-invariant theory.

Lorentz-violating quantum electrodynamics can be constructed by coupling the free photon described by the Lagrange density (2.1) to a standard spin- $\frac{1}{2}$ Dirac particle $f$ with electric charge $e_{f}$ and inertial mass $M_{f}$,

$$
\begin{aligned}
\mathcal{L}_{\text {modQED }} & =\mathcal{L}_{\text {modMaxwell }}+\mathcal{L}_{\text {Dirac }} \\
\mathcal{L}_{\text {Dirac }} & =\bar{\psi}\left[\gamma^{\mu}\left(i \partial_{\mu}-e_{f} A_{\mu}\right)-M_{f}\right] \psi
\end{aligned}
$$

The Lorentz-violating operators with the symmetry structure used for the isotropic modified Maxwell theory can also be moved into the fermion sector by an appropriate change of spacetime coordinates [3], but, for definiteness, we keep the theory as defined by (2.7). The quantum theory corresponding to (2.7) has been studied in Ref. [11], in particular as regards microcausality and unitarity.

For negative values of $\kappa$, the photon becomes unstable and can produce other particles by the decay process $\widetilde{\gamma} \rightarrow f+\bar{f}$, where $f \bar{f}$ corresponds to a generic pair of electrically charged fermions and $\widetilde{\gamma}$ denotes the "photon" from the isotropic modified Maxwell theory (the massless spin-1 particle $\widetilde{\gamma}$ has, in particular, nonstandard polarization vectors, see below). This process is called photon decay $(\mathrm{PhD})$. For positive values of $\kappa$, photons can be emitted by an electrically charged fermion $f$ and the process has been called vacuum Cherenkov (VCh) radiation, $f \rightarrow f+\widetilde{\gamma}$. 
The exact tree-level calculations of the vacuum-Cherenkov and photon decay processes have been presented in Ref. [12], with certain technical details collected in Ref. [13]. (The corresponding effects in a CPT-odd photonic theory coupled to scalar and spinor electrodynamics have also been studied $[14,15]$.) The absence of experimental evidence for these two nonstandard decay processes has been used in the past to put constraints on $\kappa$. Precise measurements of the energy loss of electrons in particle colliders have been considered for determining an indirect laboratory bound on $|\kappa|$ of order $10^{-11}$ [16] and the precise knowledge of the synchrotron loss rate can improve this bound to the $5 \times 10^{-15}$ level [17]. But an even tighter two-sided bound follows from using observations of high-energy gamma rays and cosmic rays in the Earth's atmosphere [12],

$$
-9 \times 10^{-16}<\kappa<6 \times 10^{-20}
$$

which holds at the two- $\sigma$ level $(98 \%$ CL). Another indirect lower bound [18],

$$
\kappa \gtrsim-6 \times 10^{-20}
$$

can be obtained by making an appropriate coordinate transformation to move the isotropic Lorentz violation from the photon sector into the matter sector (in particular, the electron sector) and by using the observed synchrotron radiation from high-energy electrons in the Crab Nebula. Specifically, the lower bound (2.10) results from Eq. (26) in Ref. [18] and the following isotropic Lorentz-violating parameters: $c_{X X}=c_{Y Y}=c_{Z Z}=-\kappa$ and $c_{\mu \nu}=0$ otherwise (note that these $c_{\mu \nu}$ are not the commonly used traceless-symmetric parameters, cf. Ref. [19]). The astrophysical bound (2.10) is, however, only qualitative, as Ref. [18] does not give a confidence level.

In most of the literature, the particles involved in these reactions have been considered to be structureless (pointlike). The description of photon decay into Dirac fermion-antifermion pairs as well as vacuum Cherenkov radiation by a Dirac fermion is appropriate for truly elementary particles such as electrons. But, for bounds obtained from particle showers in the Earth's atmosphere, we need a careful treatment of the internal structure of the primary hadron.

In this work, we extend the analysis of Ref. [12] by considering vacuum Cherenkov radiation emitted by a realistic proton at high energies, relevant for ultra-high-energy cosmic rays. For completeness, we also discuss the decay of an energetic astrophysical photon into an electron-positron pair, relevant for observations made with gamma-ray telescopes. The somewhat more academic case of photon decay into a proton-antiproton pair is discussed in App. A.

\section{VACUUM CHERENKOV RADIATION}

For a positive Lorentz-violating parameter $\kappa$ in the theory $(2.7)$, the maximum attainable velocity of a charged particle $(c=1)$ can be larger than the photon phase velocity from (2.6). The Cherenkov-like emission of a photon by a proton of charge $e_{p} \equiv e$ is then already possible in the vacuum. The modifications introduced by $\kappa$ to the photon polarization vectors $\tilde{\varepsilon}_{\mu}^{(\lambda)}$ are obtained by solving the equation of motion $(2.2)$ for the gauge field $A_{\mu}$, with proper normalization factors from the quantum theory $[11,13]$.

The averaged squared amplitude for a photon of energy $\omega$ emitted by an electrically 
charged Dirac fermion with proton mass $M_{p} \equiv M$ and energy $E$ has the form

$$
\begin{aligned}
\overline{\left|\mathcal{M}_{\mathrm{VCh}}\right|^{2}}= & \frac{e^{2}}{2} \sum_{\lambda} \sum_{s, s^{\prime}} \mid \bar{u}_{s^{\prime}}\left(p^{\prime}\right) \gamma^{\mu} u_{s}(p) \tilde{\varepsilon}_{\mu}^{\left.(\lambda) *\right|^{2}} \\
= & \frac{32 \pi \alpha|\kappa|}{(1+\kappa)^{2}}\left[E(E-\omega)-(1+\kappa) \frac{M^{2}}{2 \kappa}\right. \\
& \left.+\frac{\omega^{2}}{2(1-\kappa)}\right],
\end{aligned}
$$

where $\alpha \equiv e^{2} /(4 \pi)$ is the fine-structure constant. The total emission rate of Cherenkov photons by a structureless charged fermion is then given by

$$
\widehat{\Gamma}^{\mathrm{VCh}}=\frac{1}{4 \pi^{2}} \frac{1}{2 E} \int \frac{d^{3} q}{2 \omega} \frac{d^{3} p^{\prime}}{2 E^{\prime}} \overline{\left|\mathcal{M}_{\mathrm{VCh}}\right|^{2}} \delta^{4}\left(p-p^{\prime}-q\right)
$$

with $p_{0}^{\prime}=E^{\prime}=\sqrt{\left|\boldsymbol{p}^{\prime}\right|^{2}+M^{2}}$ and $q_{0}=\omega=\omega(|\boldsymbol{q}|)$ as defined by (2.6). From now on, the 'hat' on $\Gamma$ and similar quantities signifies that we are considering pointlike particles. Inserting a factor $\omega$ into the integrand and performing the necessary phase-space integrals produces the Cherenkov power radiated by a pointlike "proton" of energy $E$,

$$
\begin{aligned}
\widehat{P}(E)= & \frac{\alpha}{12 \kappa^{3} E \sqrt{E^{2}-M^{2}}}\left(\sqrt{E^{2}-M^{2}}-E / n\right)^{2} \\
\times & {\left[2 E^{2}\left(2 \kappa^{2}+4 \kappa+3\right)\right.} \\
& -3 M^{2}(1+\kappa)(1+2 \kappa) \\
& \left.-2 n E \sqrt{E^{2}-M^{2}}(1-\kappa)(4 \kappa+3)\right] .
\end{aligned}
$$

Expression (3.3) reduces to Eq. (8) of Ref. [12].

If the fermion producing the Cherenkov emission is a realistic proton, the internal structure becomes relevant at high energies, which is the case for proton primaries in energetic cosmic rays. Considering the proton as a composite particle, the Cherenkov photon can be taken as emitted by the charged partons (quarks) in the proton rather than by the proton as a whole (Fig. 1). Kinematically, the Cherenkov emission can occur when a proton of mass $M$ has an energy above the threshold

$$
E_{\mathrm{th}}=M \sqrt{\frac{1+\kappa}{2 \kappa}} .
$$

This threshold energy arises from energy-momentum conservation and is independent of the internal structure of the proton.

In this composite-proton description, Cherenkov radiation corresponds to the process $p \rightarrow X+\widetilde{\gamma}$, where $X$ stands for any hadronic final state. In fact, the photon emission and the corresponding parton recoil can lead to a hadronic final state different from the parent proton. For the description of this process, we adopt the averaged squared amplitude (3.1) for the emission of a photon by a charged parton. For a proton with energy $E>E_{\mathrm{th}}$, the Cherenkov emission rate produced by a charged parton carrying a fraction $x$ of the proton 
momentum takes the following form at tree level:

$$
\begin{aligned}
\frac{d^{2} \widehat{\Gamma}_{i}^{\mathrm{VCh}}}{d x d \omega}= & \frac{\alpha e_{i}^{2}}{E \sqrt{E^{2}-M^{2}}}\left[\frac{2 \kappa E}{1-\kappa^{2}}\left(E-\frac{\omega}{x}\right)\right. \\
& \left.-\frac{M^{2}}{1-\kappa}+\frac{\kappa}{\left(1-\kappa^{2}\right)(1-\kappa)} \frac{\omega^{2}}{x^{2}}\right],
\end{aligned}
$$

where the index $i$ denotes the parton flavor and $e_{i}$ is the parton charge in units of the proton charge $e$.

Notice that expression (3.5) allows for the identification of the Cherenkov angle. The obtained Cherenkov angle then includes the classical Huygens term $\left(\propto \omega^{0}\right)[20-23]$, the linear quantum correction $\left(\propto \omega^{1}\right)$ [24-27], and the quadratic quantum correction $\left(\propto \omega^{2}\right)$ which arises due to the fermionic nature of the radiating particle [15]. Direct calculation shows that, even though the index of refraction is nondispersive, the energy of the radiated photon has a cutoff given by

$$
\omega_{\max }=x\left(\frac{1-\kappa}{\kappa}\right)\left[\sqrt{\frac{1+\kappa}{1-\kappa}} \sqrt{E^{2}-M^{2}}-E\right] .
$$

Classically, the radiated photon energy is unbounded, leading to a divergent radiated power. The correct implementation of energy-momentum conservation relies on the photon's corpuscular nature, giving rise to a recoil of the fermion due to the photon emission [24-28]. Similarly, the expression for $\omega_{\max }$ in (3.6) is a consequence of the quantum-field-theoretic treatment of the fermion and photon [15].

The expression (3.6) of the cutoff energy for the composite proton implies that the Cherenkov emission becomes suppressed for small $x$. This suggests that the quark sea is irrelevant for Cherenkov radiation and that the process is dominated by the valence quarks. For the same reason, the decay process near threshold will primarily proceed by elastic emission of photons, that is, without modifying the structure of the parent hadron. Remark that, even though the emitted photon can have ultrahigh energies, the momentum transfer from the primary proton is suppressed by $\kappa$,

$$
Q^{2}=-q^{2}=\frac{2 \kappa \omega^{2}}{1-\kappa} \leq \frac{2 \kappa}{1-\kappa} \omega_{\max }^{2}=\mathrm{O}\left(\kappa x^{2} E^{2}\right)
$$

The quantity (3.7) corresponds to the so-called effective mass square, discussed extensively in Secs. 2 and 4 of Ref. [14] for various Lorentz-violating decays. For $E \gg E_{\mathrm{th}}, Q^{2}$ can be larger than $M^{2}$ and the photon emission can be accompanied by the creation of additional hadrons (baryon number conservation can be assumed to hold true).

The total radiated power by the composite proton $(p \rightarrow X+\widetilde{\gamma})$ is now obtained by folding the parton distribution function $f_{i}\left(x, Q^{2}\right)$ with the power radiated by the corresponding parton,

$$
P(E)=\sum_{i} \int_{0}^{1} d x \int_{0}^{\omega_{\max }} d \omega f_{i}\left(x, Q^{2}\right) \omega \frac{d^{2} \widehat{\Gamma}_{i}^{\mathrm{VCh}}}{d x d \omega},
$$

where (3.5) is to be used in the integrand and $\omega_{\max }$ and $Q^{2}$ are given by (3.6) and (3.7), respectively. The sum in (3.8) contains contributions from all charged partons in the proton. Recall that $f_{i}\left(x, Q^{2}\right)$ corresponds to the probability density that a charged parton of flavor 
$i$ carries a fraction $x$ of the longitudinal momentum, where $Q^{2}$ parameterizes the resolution scale at which the parent hadron is probed. The radiated power (3.8) includes all possible hadronic final states. In fact, expression (3.8) is analogous to the parton-model result for the total cross section of deep-inelastic scattering $e^{-}+p \rightarrow e^{-}+X$ (cf. Sec. 17.3 of Ref. [29]). From (3.8), the characteristic Cherenkov radiation length is given by

$$
l_{\mathrm{VCh}}(E) \equiv c E / P(E),
$$

with the velocity $c$ temporarily restored.

Libraries with global fits from experimental data as well as the evolution to different energy scales of the parton distribution functions (PDFs) of the proton are publicly available [30] and can be used to numerically perform the integrations in (3.8). With the PDFs from the CTEQ collaboration [31], the Cherenkov power radiated by a proton is shown in Fig. 2. In addition, we give in Fig. 2 the power radiated by a neutron, which can also be obtained approximately by the isospin transformation $u \leftrightarrow d$ on the proton PDFs and parton charges. (Incidentally, the finite lifetime of the neutron due to $\beta$-decay is irrelevant for the present discussion.) The "structureless proton" in Fig. 2 corresponds to the limit in which the proton is approximated by a pointlike Dirac fermion [12]. The corresponding Cherenkov radiation lengths are shown in Fig. 3. We emphasize that, in our parton-model calculations, the effective mass square (3.7) controls the energy scale for the PDFs rather than the energy of the incoming proton, as used in the analysis of Ref. [32].

Figure 2 shows that the energy loss characterized by the radiated power is lower for the proton than the structureless fermion. This reduction can be understood as the combination of several effects. On average, charged partons carry only half of the proton energy, while the other half is carried by gluons. Furthermore, the functional form of the power radiated by the proton is similar to that of the structureless fermion folded with the PDFs of each charged parton and with an overall factor $x^{2}$, where the momentum fraction $x f_{u}\left(x, Q^{2}\right)$ peaks around $x=0.2$ for relatively low momentum transfer. These effects give a total suppression factor of approximately 10, in agreement with the numerical result shown in Fig. 2. This suppression factor slowly increases for higher energies as the $u$-quark sea spreads the momentum fraction to lower values of $x$. For completeness, the photon emission spectrum is also given in Fig. 4 .

The detection of a cosmic-ray primary with energy $E_{\text {prim }}$ implies the condition

$$
E_{\text {prim }}<E_{\text {th }}
$$

with $E_{\text {th }}$ from (3.4) for $M=M_{\text {prim }}$. Namely, if (3.10) would not hold, the primary would have lost most of its energy on the journey through space and the Earth's atmosphere. The validity of the condition $E_{\text {prim }}<E_{\text {th }}$ is independent of the astrophysical processes involved in the creation and acceleration of the cosmic-ray primary. In fact, we can focus on the path through the Earth's atmosphere (with a height scale of order $10 \mathrm{~km}$ ), where the particle track can be observed directly (for example, by the fluorescence detectors of the Pierre Auger Observatory [33] or by gamma-ray telescopes [34]). The only assumption is that the proton propagation distance $d$ (of the order of $1 \mathrm{~km}$ or more) is significantly larger than the characteristic Cherenkov radiation length, $d \gg \widehat{l}_{\mathrm{VCh}}$. The upper bound (2.9) on the parameter $\kappa$ was obtained from (3.10) by using the detection of a 212-EeV cosmic ray by the Pierre Auger Observatory [35] and by assuming a structureless iron nucleus with $M_{\text {prim }}=52 \mathrm{GeV}[12]$. For a primary structureless proton with mass $M=0.94 \mathrm{GeV}$, the upper bound in (2.9) would be reduced by a factor $(0.94 / 52)^{2}$ to a value of $2 \times 10^{-23}$.

The reduced power radiated by a realistic proton or nucleus with respect to the structureless-fermion result indicates that this realistic particle will travel over a somewhat larger 
distance before efficiently losing energy in the form of vacuum Cherenkov radiation. As discussed above, this extra distance is about one order of magnitude more than the structureless case. Since the characteristic distance for the photon emission by a structureless fermion is only a fraction of a meter (Fig. 3), the assumption of travel distances being larger than the decay length $\left(d \gg l_{\mathrm{VCh}}\right)$ is completely justified, even for the realistic proton primary described in this work (for a nucleus $N$, we simply scale the proton results with $e \rightarrow Z_{N} e$ and $\left.M \rightarrow M_{N}\right)$. These remarks imply that the upper bound on the parameter $\kappa$ as given in Ref. [12], based on the threshold condition (3.10), also holds for a realistic proton or nucleus with internal structure taken into account.

\section{PHOTON DECAY}

For a negative Lorentz-violating parameter $\kappa$ in the theory (2.7), the photon becomes unstable and the production of a pair of electrically charged fermions is kinematically allowed at sufficiently high energies (Fig. 5). The averaged squared amplitude for photon decay equals the averaged squared amplitude for vacuum Cherenkov radiation (3.1),

$$
\begin{aligned}
\overline{\left|\mathcal{M}_{\mathrm{PhD}}\right|^{2}} & =\frac{e_{f}^{2}}{2} \sum_{\lambda} \sum_{s, s^{\prime}}\left|\bar{u}_{s}(p) \gamma^{\mu} v_{s^{\prime}}\left(p^{\prime}\right) \tilde{\varepsilon}_{\mu}^{(\lambda)}\right|^{2} \\
& =\overline{\left|\mathcal{M}_{\mathrm{VCh}}\right|^{2}} .
\end{aligned}
$$

The photon decay rate into a fermion-antifermion pair then takes the form

$$
\widehat{\Gamma}^{\mathrm{PhD}}=\frac{1}{4 \pi^{2}} \frac{1}{2 \omega} \int \frac{d^{3} p}{2 E} \frac{d^{3} p^{\prime}}{2 E^{\prime}} \overline{\left|\mathcal{M}_{\mathrm{PhD}}\right|^{2}} \delta^{4}\left(q-p-p^{\prime}\right),
$$

with $p_{0}=E=\sqrt{|\boldsymbol{p}|^{2}+M_{f}^{2}}$ and similarly for $p_{0}^{\prime}=E^{\prime}$. Energy-momentum conservation implies that pair production occurs for photon energies above the threshold

$$
\omega_{\text {th }}=2 M_{f} \sqrt{\frac{1-\kappa}{-2 \kappa}},
$$

where $M_{f}$ is the equal mass of the fermion and the antifermion (note that $C P T$ and $C$ are exact symmetries of the theory considered). Above this threshold, the decay of a photon of energy $\omega$ produces a fermion with energy $E$ in the range $\left[E_{-}, E_{+}\right]$, with

$$
E_{ \pm}=\frac{\omega}{2}\left[1 \pm n \sqrt{1-\frac{\omega_{\mathrm{th}}^{2}}{\omega^{2}}}\right]
$$

where $n$ is the index of refraction (2.5). The decay constant is obtained by integrating over the available fermion energies,

$$
\widehat{\Gamma}^{\mathrm{PhD}}=\int_{E_{-}}^{E_{+}} d E \frac{d \widehat{\Gamma}^{\mathrm{PhD}}}{d E} .
$$

Specializing to the case of an electron-positron pair (with charges $e_{f}= \pm e$ and mass $M_{f}=M_{e} \equiv m$ ) and performing the energy integral in (4.5) then gives

$$
\widehat{\Gamma}^{\mathrm{PhD}}(\omega)=\frac{\alpha}{3} \frac{-\kappa}{1-\kappa^{2}} \omega \sqrt{1-\omega_{\mathrm{th}}^{2} / \omega^{2}}\left[2+\omega_{\mathrm{th}}^{2} / \omega^{2}\right],
$$


where $\alpha \equiv e^{2} /(4 \pi)$ is the fine-structure constant and $\omega_{\text {th }}$ is given by (4.3) in terms of the electron mass $m$. Expression (4.6) is equivalent to Eq. (11) of Ref. [12]. The characteristic decay length determined by the photon lifetime is defined as follows:

$$
\widehat{l}_{\mathrm{PhD}}(\omega) \equiv c / \widehat{\Gamma}^{\mathrm{PhD}}(\omega),
$$

with the velocity $c$ temporarily restored.

Similar to the case of vacuum Cherenkov radiation, the photon momentum transfer is suppressed by the parameter $\kappa$, which implies that $Q^{2}=q^{2}$ is significant only for large values of the photon energy. In fact, expression (3.7) gives

$$
Q^{2}=\left(2 m \omega / \omega_{\text {th }}\right)^{2},
$$

with $\omega_{\text {th }}$ given by (4.3) in terms of the electron mass $m$. Again, the quantity (4.8) plays the role of the effective mass square entering a Lorentz-violating decay [14].

The energy scale entering $Q^{2}$ from (4.8) is given by the mass $2 m$ of the electron-positron pair for photon energies close to the threshold. At these low momentum transfers, the electron is a point particle to very high precision: compositeness bounds for the electron are at multi-TeV energy scales (cf. Ref. [36], pp. 1631-1636). In short, the point-particle calculation for photon decay into an electron-positron pair is completely reliable. The results are shown in Figs. 6 and 7. For completeness, the electron emission spectrum is also given in Fig. 8.

Following the same reasoning as for the case of vacuum Cherenkov radiation, the observation of a primary photon with energy $\omega_{\gamma, \text { prim }}$ implies the condition

$$
\omega_{\gamma, \text { prim }}<\omega_{\mathrm{th}}
$$

with $\omega_{\text {th }}$ given by (4.3) in terms of the electron mass $m=511 \mathrm{keV}$. Namely, if (4.9) would not hold, the photon would have decayed along the journey through space and the Earth's atmosphere. Once again, the validity of this condition is independent of the astrophysical processes involved in the creation of these gamma-ray photons and the only assumption is that the photons have traveled a distance $d$ larger than the characteristic decay length (4.7). The lower bound (2.9) on the parameter $\kappa$ was obtained from (4.9) by using the detection of 30-TeV gamma rays by the High Energy Stereoscopic System (HESS) [37] and by assuming

the decay of photons into electron-positron pairs [12]. In this case, the decay length of a $30-\mathrm{TeV}$ photon would be a few millimeters (Fig. 7), so that the assumption $d \gg \widehat{l}_{\mathrm{PhD}}$ is unquestionably valid.

\section{SUMMARY}

In this article, we have studied two nonstandard decay process in CPT-even Lorentzviolating quantum electrodynamics. In particular, we have considered the effects of an isotropic nonbirefringent modification leading to vacuum Cherenkov radiation by a proton and photon decay into an electron-positron pair. Previous studies [12] assumed structureless particles for both processes.

For the first process, vacuum Cherenkov radiation by a proton in the theory (2.7) with $\kappa>0$, we have now also performed a calculation in the framework of the parton model (higher-order QCD corrections have not been considered). The Cherenkov power is found 
to be reduced by approximately one order of magnitude but the threshold energy remains unchanged compared to the point-particle calculation. For the second process, photon decay into an electron-positron pair in the theory (2.7) with $\kappa<0$, the point-particle calculation still holds and we have given more details than available in the literature up till now, in particular the momentum transfer $Q^{2}$ and the photon decay length. The upshot is that the previous two-sided bound (2.9) from Ref. [12] remains unchanged.

The multimessenger astrophysics program studying high-energy phenomena with ultrahigh-energy cosmic rays [32, 38, 39], cosmic gamma rays [40], and cosmic neutrinos [41] has developed the field of astroparticle physics over the last years and now serves as a powerful tool to test fundamental physics symmetries.

\section{ACKNOWLEDGMENTS}

It is a pleasure to thank M. Risse for discussions and the referee for useful comments. The start-up phase of this work was supported in part by the "Helmholtz Alliance for Astroparticle Physics HAP," funded by the Initiative and Networking Fund of the Helmholtz Association, and the main phase was supported in part by the German Research Foundation (DFG) under Grant No. KL 1103/4-1.

\section{Appendix A: Photon decay into a proton-antiproton pair}

A direct parton-model calculation of the Lorentz-violating decay process $\widetilde{\gamma} \rightarrow p+\bar{p}$ appears to be difficult if we wish to use standard fragmentation functions [36]. Instead, we will take a different approach employing electromagnetic form factors (see, e.g., Ref. [42] for a review).

The standard pointlike Dirac interaction term from (2.8) is to be generalized by the introduction of form factors $F_{n}$. Going to momentum space, the interaction term between protons $(p)$, antiprotons $(\bar{p})$, and modified photons $(\widetilde{\gamma})$ then becomes

$$
\begin{aligned}
\mathcal{L}_{\text {proton }}^{(\mathrm{int})}= & \bar{\psi}\left(p^{\prime}\right) \text { e } A_{\mu}(q)\left[\gamma^{\mu} F_{1}\left(q^{2}\right)+i \sigma^{\mu \nu} q_{\nu} /(2 M) F_{2}\left(q^{2}\right)\right. \\
& +\ldots] \psi(p) \delta^{4}\left(p^{\prime}-q-p\right)
\end{aligned}
$$

The ellipsis allows for the possibility that this Lorentz-violating theory needs further terms.

As a first approximation, we will only use the $F_{1}$ form factor,

$$
\begin{aligned}
& F_{1}=\widetilde{F}, \\
& F_{n}=0, \text { for } n \geq 2 .
\end{aligned}
$$

In the $\kappa>0$ theory (2.7) with form-factor interactions (A1) and Ansätze (A2), the Cherenkov power becomes

$$
\widetilde{P}^{\mathrm{VCh}}(E)=\int_{0}^{\omega_{\max }} d \omega \omega\left|\widetilde{F}\left(-\frac{2 \kappa \omega^{2}}{1-\kappa}\right)\right|^{2} \frac{d \widehat{\Gamma}^{\mathrm{VCh}}}{d \omega},
$$

with $\omega_{\max }$ given by (3.6) for $x=1$ and $d \widehat{\Gamma}^{\mathrm{VCh}} / d \omega$ given by the right-hand side of (3.5) for $x=1$ and $e_{i}^{2}=1$. Here and in the following, the tilde on a quantity denotes the use of the 
form factors (A2). Without form factor $F_{2}$, the expression for photon decay in the $\kappa<0$ theory is relatively simple,

$$
\widetilde{\Gamma}^{\mathrm{PhD}}(\omega)=\left|\widetilde{F}\left(-\frac{2 \kappa \omega^{2}}{1-\kappa}\right)\right|^{2} \widehat{\Gamma}^{\mathrm{PhD}}(\omega),
$$

where $\widehat{\Gamma}^{\mathrm{PhD}}$ is given by (4.6).

For the theory with $\kappa=6 \times 10^{-20}$, the direct parton-model results of the Cherenkov power $P$ as shown in Fig. 2 are reproduced by inserting $\widetilde{F} \sim \sqrt{1 / 20}$ into the integrand of (A3). For timelike $q^{2}>0$, relevant to photon decay in the $\kappa<0$ theory, the following simple approximation can be used:

$$
\widetilde{F}\left(q^{2}\right) \sim \frac{\mathrm{GeV}^{4}}{\left(q^{2}\right)^{2}+\mathrm{GeV}^{4}},
$$

whose physics motivation will be given shortly. For the theory with $\kappa=-6 \times 10^{-20}$, the explicit function (A5) inserted in (A4) then gives the results shown in Fig. 9. Incidentally, the same function (A5) for spacelike $q^{2}<0$ inserted into the integrand of (A3) in the $\kappa>0$ theory gives a constant value for $\widetilde{P}^{\mathrm{VCh}}(E)$ as $E \rightarrow \infty$. For the parameter values of Fig. 2, this asymptotic $\widetilde{P}^{\text {VCh }}$ value is approximately $3 \times 10^{-3} \mathrm{GeV}^{2}$, but note that this form-factor result only concerns elastic photon emission, whereas the initial-proton parton-model curve in Fig. 2 refers to the inclusive process.

The promised background on the choice (A5) is as follows. In terms of Sachs form factors $G_{E}\left(q^{2}\right) \equiv F_{1}\left(q^{2}\right)+\left[q^{2} /\left(4 M^{2}\right)\right] F_{2}\left(q^{2}\right)$ and $G_{M}\left(q^{2}\right) \equiv F_{1}\left(q^{2}\right)+F_{2}\left(q^{2}\right)$, a common working hypothesis is $\left|G_{E}\right|=\left|G_{M}\right|=G_{\text {eff. }}$ Considering real form factors (appropriate for $\left|q^{2}\right| \rightarrow \infty$ ), this hypothesis corresponds precisely to our assumption (A2), with $F_{1}=G_{\text {eff }}$ and $F_{2}=0$, so that $(\mathrm{A} 4)$ can be read as $\left|G_{\text {eff }}\right|^{2} \times \widehat{\Gamma}^{\mathrm{PhD}}$. The experimental data for $G_{\text {eff }}\left(q^{2}\right)$ with timelike $q^{2}$ between approximately $4 \mathrm{GeV}^{2}$ and $30 \mathrm{GeV}^{2}$ (see Fig. 10 of Ref. [42]) is very roughly and qualitatively fitted by the simple function (A5) with the expected asymptotic behavior for $\left|q^{2}\right| \rightarrow \infty$, leaving aside the precise behavior at the physical threshold $\left(q^{2}\right)_{\text {phys }}=4 M^{2} \approx$ $3.53 \mathrm{GeV}^{2}$.

Returning to the photon-decay process of Fig. 9, the question is where the definitive curve for a realistic proton lies. We expect the definitive curve for the inclusive process $\widetilde{\gamma} \rightarrow$ $p+\bar{p}+X$ to lie closer to the structureless curve than to the form-factor curve. Heuristically we can give the following argument. In the modified QED model (2.7) with form factors (A1), on the one hand, the photon $\widetilde{\gamma}$ directly creates a complete extended proton particle and a complete extended antiproton particle. In the parton-model version, on the other hand, the photon $\widetilde{\gamma}$ first creates a pointlike antiquark and a pointlike quark, and then dresses them by pulling (anti)quarks and gluons out of the vacuum, possibly creating more hadrons than a single proton-antiproton pair. For relatively large momentum transfer $\left(q^{2} \gtrsim \mathrm{GeV}^{2}\right)$, it seems that the parton-model description is more appropriate, but the definitive calculation remains to be performed. It may also be instructive to compare to the case of $p \bar{p}$ production in electron-positron annihilation, for which many experimental results exist (cf. Sec. 20.6 in Ref. [36]). 
[1] S. Chadha and H.B. Nielsen, "Lorentz invariance as a low-energy phenomenon," Nucl. Phys. B 217, 125 (1983).

[2] V.A. Kostelecký and M. Mewes, "Signals for Lorentz violation in electrodynamics," Phys. Rev. D 66, 056005 (2002), arXiv:hep-ph/0205211.

[3] Q. Bailey and V.A. Kostelecký, "Lorentz-violating electrostatics and magnetostatics," Phys. Rev. D 70, 076006 (2004), arXiv:hep-ph/0407252.

[4] S.M. Carroll, G.B. Field, and R. Jackiw, "Limits on a Lorentz and parity violating modification of electrodynamics," Phys. Rev. D 41, 1231 (1990).

[5] S.M. Carroll and G.B. Field, "Is there evidence for cosmic anisotropy in the polarization of distant radio sources?," Phys. Rev. Lett. 79, 2394 (1997), arXiv:astro-ph/9704263.

[6] V.A. Kostelecký and M. Mewes, "Cosmological constraints on Lorentz violation in electrodynamics," Phys. Rev. Lett. 87, 251304 (2001), arXiv:hep-ph/0111026.

[7] H.E. Ives and G.R. Stilwell, "An experimental study of the rate of a moving atomic clock," J. Opt. Soc. Am. 28, 215 (1938).

[8] S. Reinhardt et al., "Test of relativistic time dilation with fast optical atomic clocks at different velocities," Nature Phys. 3, 861 (2007).

[9] F.N. Baynes, M.E. Tobar, and A.N. Luiten, "Oscillating test of the isotropic shift of the speed of light," Phys. Rev. Lett. 108, 260801 (2012).

[10] Y. Michimura et al., "New limit on Lorentz violation using a double-pass optical ring cavity," Phys. Rev. Lett. 110, 200401 (2013), arXiv:1303.6709.

[11] F.R. Klinkhamer and M. Schreck, "Consistency of isotropic modified Maxwell theory: Microcausality and unitarity," Nucl. Phys. B 848, 90 (2011), arXiv:1011.4258.

[12] F.R. Klinkhamer and M. Schreck, "New two-sided bound on the isotropic Lorentz-violating parameter of modified Maxwell theory," Phys. Rev. D 78, 085026 (2008), arXiv:0809.3217.

[13] C. Kaufhold, F.R. Klinkhamer, and M. Schreck, "Tree-level calculation of vacuum Cherenkov radiation in isotropic nonbirefringent modified-Maxwell theory," report KA-TP-32-2007, http://www.itp.kit.edu.

[14] C. Kaufhold and F.R. Klinkhamer, "Vacuum Cherenkov radiation and photon triple-splitting in a Lorentz-noninvariant extension of quantum electrodynamics," Nucl. Phys. B 734, 1 (2006), arXiv:hep-th/0508074.

[15] C. Kaufhold and F.R. Klinkhamer, "Vacuum Cherenkov radiation in spacelike MaxwellChern-Simons theory," Phys. Rev. D 76, 025024 (2007), arXiv:0704.3255.

[16] M.A. Hohensee, R. Lehnert, D.F. Phillips, and R.L. Walsworth, "Particle-accelerator constraints on isotropic modifications of the speed of light," Phys. Rev. Lett. 102, 170402 (2009), arXiv:0904.2031.

[17] B. Altschul, "Bounding isotropic Lorentz violation using synchrotron losses at LEP," Phys. Rev. D 80, 091901 (2009), arXiv:0905.4346.

[18] B. Altschul, "Lorentz violation and synchrotron radiation," Phys. Rev. D 72, 085003 (2005), arXiv:hep-th/0507258.

[19] B. Altschul, "Synchrotron and inverse Compton constraints on Lorentz violations for electrons," Phys. Rev. D 74, 083003 (2006), arXiv:hep-ph/0608332.

[20] P.A. Cherenkov, "The visible glow of pure liquids under the action of $\gamma$-rays," Dokl. Akad. Nauk Ser. Fiz. 2, 451 (1934). 
[21] S.I. Vavilov, "On the possible causes of the blue $\gamma$-glow in liquids," Dokl. Akad. Nauk Ser. Fiz. 2, 457 (1934).

[22] P.A. Cherenkov, "Visible radiation produced by electrons moving in a medium with velocities exceeding that of light," Phys. Rev. 52, 378 (1937).

[23] I.M. Frank and I.E. Tamm, "Coherent visible radiation of fast electrons passing through matter," Dokl. Akad. Nauk Ser. Fiz. 14, 109 (1937).

[24] V.L. Ginzburg, "Quantum theory of light radiation from an electron moving uniformly in a medium," Zh. Eksp. Teor. Fiz. 10, 589 (1940).

[25] R.T. Cox, "Momentum and energy of photon and electron in the Čerenkov radiation," Phys. Rev. 66, 106 (1944).

[26] J.M. Jauch and K M. Watson, "Phenomenological quantum electrodynamics. Part II: Interaction of the field with charges," Phys. Rev. 74, 1485 (1948).

[27] V.L. Ginzburg, "Radiation by uniformly moving sources (Vavilov-Cherenkov effect, transition radiation, and other phenomena)," Phys. Usp. 39, 973 (1996).

[28] B.D. Altschul, "Finite duration and energy effects in Lorentz-violating vacuum Cerenkov radiation," Nucl. Phys. B 796, 262 (2008), arXiv:0709.4478.

[29] M.E. Peskin and D.V. Schroeder, An Introduction to Quantum Field Theory (Addison-Wesley, Reading, MA, 1995).

[30] A. Buckley et al., "LHAPDF6: Parton density access in the LHC precision era," Eur. Phys. J. C 75, 132 (2015), arXiv:1412.7420; LHAPDF Documentation (version 6.1.5), https://lhapdf .hepforge.org/.

[31] H.L. Lai et al., "New parton distributions for collider physics," Phys. Rev. D 82, 074024 (2010), arXiv:1007.2241; http://users.phys.psu.edu/ cteq/\#PDFs.

[32] O. Gagnon and G.D. Moore, "Limits on Lorentz violation from the highest energy cosmic rays," Phys. Rev. D 70, 065002 (2004), arXiv:hep-ph/0404196.

[33] J. Abraham et al. (Pierre Auger Collaboration), "The fluorescence detector of the Pierre Auger Observatory," Nucl. Instrum. Meth. A 620, 227 (2010), arXiv:0907.4282.

[34] T. Weekes, Very High Energy Gamma-Ray Astronomy (IOP, Bristol, UK, 2003).

[35] J. Abraham et al. (Pierre Auger Collaboration), "An upper limit to the photon fraction in cosmic rays above $10^{19}-\mathrm{eV}$ from the Pierre Auger Observatory," Astropart. Phys. 27, 155 (2007), arXiv:astro-ph/0606619.

[36] K.A. Olive et al. (Particle Data Group), "The review of particle physics," Chin. Phys. C 38, 090001 (2014).

[37] F. Aharonian et al. (HESS Collaboration), "Primary particle acceleration above $100 \mathrm{TeV}$ in the shell-type Supernova Remnant RX J1713.7-3946 with deep H.E.S.S. observations," Astron. Astrophys. 464, 235 (2007), arXiv:astro-ph/0611813.

[38] F.R. Klinkhamer and M. Risse, "Ultra-high-energy cosmic-ray bounds on nonbirefringent modified-Maxwell theory," Phys. Rev. D 77, 016002 (2008), arXiv:0709.2502.

[39] F.R. Klinkhamer and M. Risse, "Addendum: Ultrahigh-energy cosmic-ray bounds on nonbirefringent modified-Maxwell theory," Phys. Rev. D 77, 117901 (2008), arXiv:0806.4351.

[40] M. Daniel (CTA Consortium Collaboration), "Lorentz invariance violation with gamma rays," arXiv:1501.00824.

[41] J.S. Díaz, V.A. Kostelecký, and M. Mewes, "Testing relativity with high-energy astrophysical neutrinos," Phys. Rev. D 89, 043005 (2014), arXiv:1308.6344.

[42] S. Pacetti, R. Baldini Ferroli, and E. Tomasi-Gustafsson, "Proton electromagnetic form factors: Basic notions, present achievements and future perspectives," Phys. Rept. 550-551, 1 (2015). 


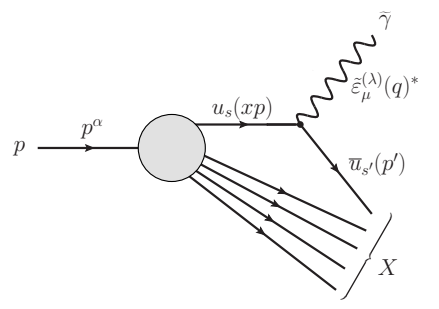

FIG. 1. Tree-level Feynman diagram for vacuum Cherenkov radiation by a charged parton inside a high-energy proton $p$. Lorentz-violating effects are contained in the modified polarization vector of the outgoing photon $\widetilde{\gamma}$ and its modified dispersion relation (2.6). For energies just above threshold, there is elastic photon emission, $p \rightarrow p+\widetilde{\gamma}$. But, for energies significantly above threshold, also inelastic photon emission processes occur, with additionally produced hadrons. The present article considers the inclusive process, $p \rightarrow X+\widetilde{\gamma}$.

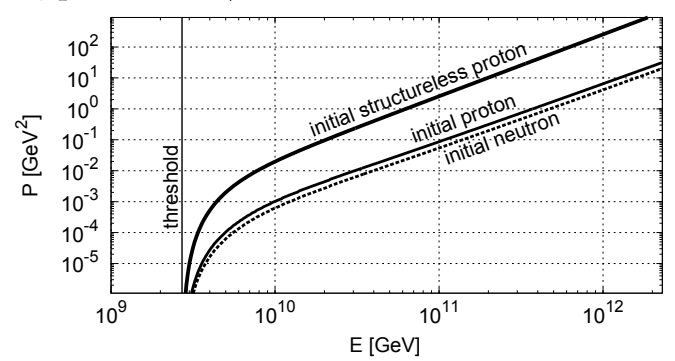

FIG. 2. Cherenkov power $P$ for the inclusive decay process $h \rightarrow X+\widetilde{\gamma}$ as a function of the energy $E$ of the initial hadron $h$, which can be a proton, a neutron, or a structureless charged Dirac fermion (the initial structureless fermion remains unchanged by the photon emission). The proton and neutron results are obtained from the parton-model expression (3.8) and further details are given in the main text. The structureless-proton result follows from (3.3). The Lorentz-violating parameter is $\kappa=6 \times 10^{-20}$ and the threshold energy for the proton case is given by Eq. (3.4).

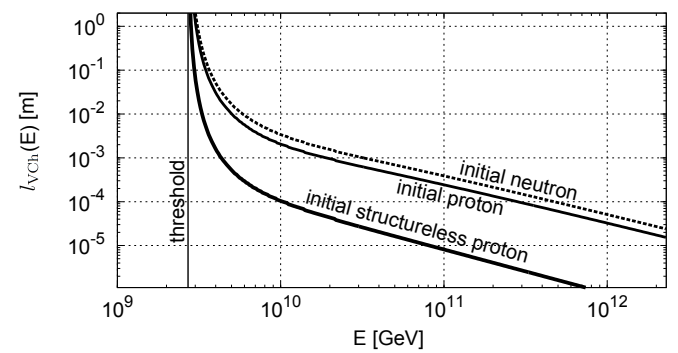

FIG. 3. Cherenkov radiation length $l_{\mathrm{VCh}}$ in meters for a proton, a neutron, and a structureless charged Dirac fermion with $\kappa=6 \times 10^{-20}$ as in Fig. 2 . The radiation length $l_{\mathrm{VCh}}$ is defined by (3.9).

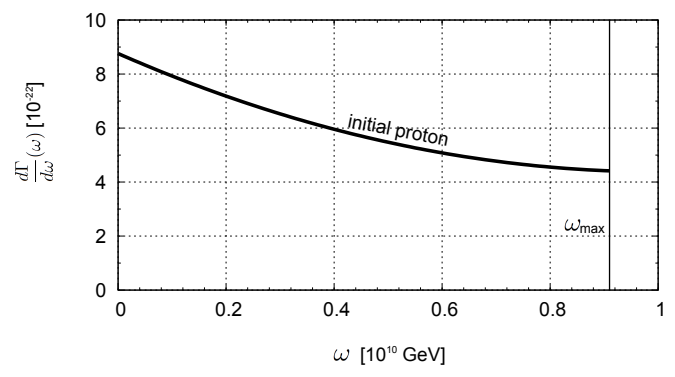

FIG. 4. Differential photon spectrum $d \Gamma / d \omega$ from vacuum Cherenkov radiation by a proton of energy $E=10^{10} \mathrm{GeV}$ in the $\kappa=6 \times 10^{-20}$ theory, as in Fig. 2. The differential photon spectrum is obtained from the parton-model expression (3.8) by omitting the $\omega$ factor in the integrand and not performing the integral over $\omega$. 


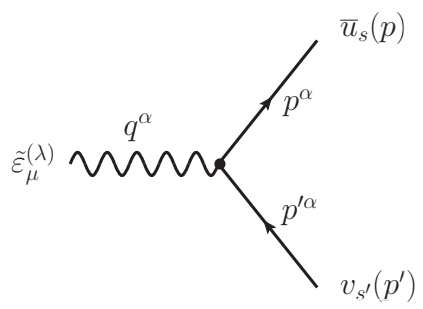

FIG. 5. Tree-level Feynman diagram for photon decay into an electron-positron pair, $\widetilde{\gamma} \rightarrow e^{-}+e^{+}$. Lorentz-violating effects are contained in the modified polarization vector and dispersion relation of the incoming photon.

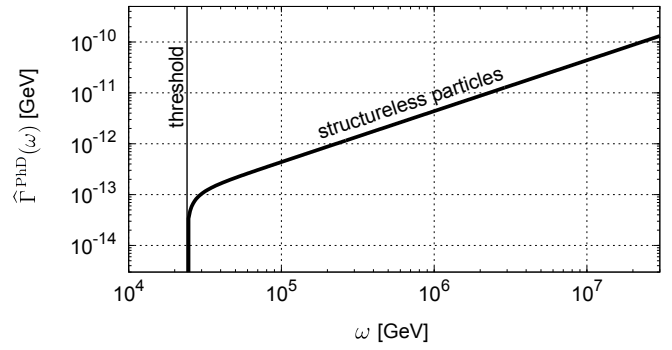

FIG. 6. Photon decay constant $\widehat{\Gamma}$ for $\widetilde{\gamma} \rightarrow e^{-}+e^{+}$as a function of the photon energy $\omega$. The pointparticle decay constant $\widehat{\Gamma}$ is given by (4.6). The Lorentz-violating parameter is $\kappa=-9 \times 10^{-16}$ and the threshold energy is given by Eq. (4.3) in terms of the electron mass.

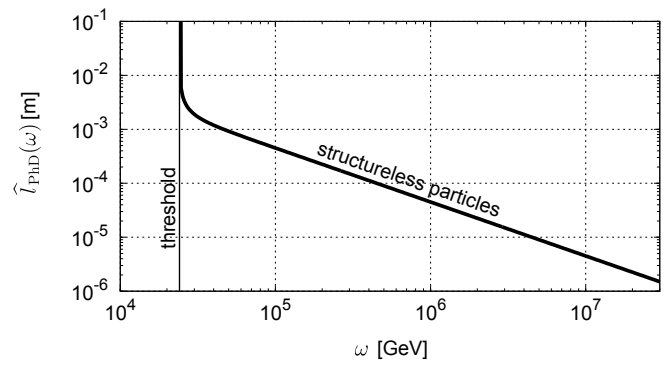

FIG. 7. Photon decay length $\widehat{l}_{\mathrm{PhD}}$ in meters for $\widetilde{\gamma} \rightarrow e^{-}+e^{+}$with $\kappa=-9 \times 10^{-16}$ as in Fig. 6 . The decay length $\widehat{l}_{\mathrm{PhD}}$ is defined by (4.7).

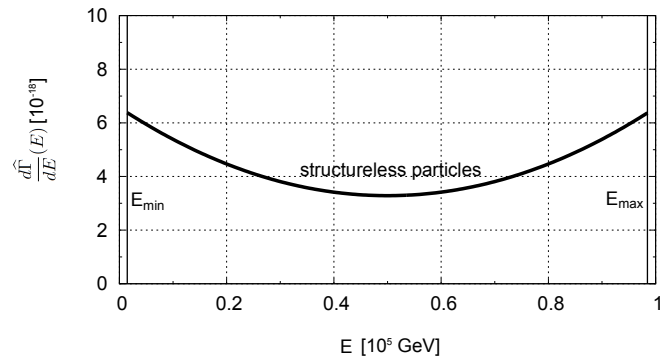

FIG. 8. Differential electron spectrum $d \widehat{\Gamma} / d E$ from the Lorentz-violating decay of a photon $\widetilde{\gamma}$ of energy $\omega=10^{5} \mathrm{GeV}$ into an electron-positron pair, calculated for pointlike particles in the $\kappa=-9 \times 10^{-16}$ theory, as in Fig. 6 . 


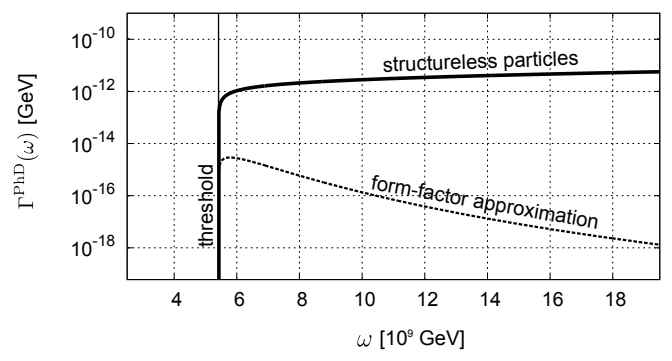

FIG. 9. Photon decay constant $\Gamma^{\mathrm{PhD}}$ for $\widetilde{\gamma} \rightarrow p+\bar{p}$ as a function of the photon energy $\omega$. Shown are the results from a structureless description and from a form-factor approximation (A1)-(A2) based on the function (A5). The Lorentz-violating parameter is $\kappa=-6 \times 10^{-20}$ and the threshold energy is given by Eq. (4.3) in terms of the proton mass. 